higher rates of self-harm than heterosexuals. Conversely, all studies of sexual orientation and completed suicide have concluded that gay men and lesbians do not die by suicide at a higher rate than heterosexuals.

Spencer (1959) followed 100 Oxford undergraduates referred by their general practitioners. Relative to $35 \%$ of controls $(n=100)$, a significantly greater proportion of patients $(51 \%)$ had homosexual behaviour, fantasies or desires. 'No patient was lost by suicide' but 9 of 10 who attempted suicide were 'persistently homosexual' (pp. 402-403). Cohen (1961) found only one same-sex couple $(1.7 \%)$ among 58 completed suicide pacts. O'Hara (1963) found only $4 \%$ lesbians and gay men in a 1-year incidence study of double suicides in Japan. Rich et al (1986) reported that $13(11 \%)$ of 119 males aged $21-42$ who died by suicide in Los Angeles had disclosed a homosexual identity prior to death. In New York City, Shaffer et al (1995) found that in $3(2.5 \%)$ of 120 completed youth (aged $\leqslant 20$ years) suicide cases the individual was gay. However, they found no gay or lesbian young people among 147 living controls matched for age, gender and ethnicity.

Thus, contrary to King et al's assertion, at least five peer-reviewed studies of sexual orientation and completed suicide have been published.

Cohen, J. (1961) A study of suicide pacts. Medico-Lega Journal, 29, |44-|5|.

King, M., McKeown, E., Warner, J., et al (2003)

Mental health and quality of life of gay men and lesbians in England and Wales. Controlled, cross-sectional study. British Journal of Psychiatry, 183, 552-558.

O'Hara, K. (1963) Characteristics of suicides in Japan, especially of parent-child double suicide. American Journal of Psychiatry, 120, 382-385.

Rich, C. L., Fowler, R. C., Young, D., et al (1986) San Diego suicide study: comparison of gay to straight males. Suicide and Life-Threatening Behavior, 16, 448-457.

Shaffer, D., Fisher, P., Hicks, R. H., et al (1995) Sexua orientation in adolescents who commit suicide. Suicide and Life-Threatening Behavior, 25 (suppl.), 64-7I.

Spencer, S. J. G. (1959) Homosexuality among Oxford undergraduates. Journal of Mental Science, 105, 393-405.

R. M. Mathy University of Minnesota Medical School, 809 Spring St NE \# 105, Minneapolis, MN 554I3-2347, USA. E-mail: math5577@umn.edu

\section{Occupational psychiatry}

In their editorial on work and employment for people with psychiatric illness,
Boardman et al (2003) overlook an important group of patients with mental ill health who are not 'mental health service users', yet who experience difficulty coping in the modern workplace. Occupational physicians are seeing an increasing number of patients with mental ill health, and a national surveillance scheme recently reported that, along with musculoskeletal symptoms, mental ill health is among the commonest reasons for consultation (see http://www.coeh.man.ac.uk/thor/opra.htm). Furthermore, mental ill health is responsible for a large proportion of early retirements due to ill health (Poole, 1997) and a large proportion of incapacity benefits are currently being paid for medically unexplained illnesses (Waddell, 2002).

Much of the burden of occupational ill health is managed in primary care, but overburdened general practitioners may miss the psychological or workplace components in these patients. To make matters worse, current psychiatric practice is dominated by 'serious' mental illness such as schizophrenia and 'dual diagnosis' patients, to the exclusion of patients with 'minor' mental illnesses such as anxiety, depression and the functional disorders. Yet it is these latter conditions that are commonly being seen in the workplace, in primary care and in those on state benefits by doctors who have little training in mental illness. Unfortunately, some psychiatrists do not receive adequate training in the management of these disorders (Bass et al, 2001), in part because they are presenting in locations outside of psychiatric services (Henderson et al, 2001). Good evidence exists that these illnesses can be treated effectively using, for example, cognitive-behavioural therapy and interpersonal therapy (Creed et al, 2003). A key feature of these studies is that the best results are usually achieved at the site where the patient presents, which is likely to be outside the province of the community mental health team.

We believe that there is a lack of expertise in the management of occupational mental ill health at its site of presentation. Psychiatrists need to engage with occupational physicians to improve the diagnosis and management of patients with psychiatric illnesses that are preventing them from working. There is also a need for more collaborative training in occupational psychiatry for psychiatrists, occupational physicians and general practitioners. Such training should be integrated into the syllabuses of all three professional groups. A diploma in occupational psychiatry might be very popular.

Bass, C., Peveler, R. \& House, A. (2001) Somatoform disorders: severe psychiatric illnesses neglected by psychiatrists. British Journal of Psychiatry, 179, II-14.

Boardman, J., Grove, B., Perkins, R., et al (2003)

Work and employment for people with psychiatric disabilities. British Journal of Psychiatry, 182, 467-468.

Creed, F., Fernandes, L., Guthrie, E., et al (2003) The cost-effectiveness of psychotherapy and paroxetine for severe irritable bowel syndrome. Gastroenterology, 124 $303-317$

Henderson, M., Holland-Elliott, K., Hotopf, M., et al (200I) Liaison psychiatry and occupational health Occupational Medicine, 5I, 479-48I.

Poole, C. J. M. (1997) Retirement on grounds of ill health: cross sectional survey in six organisations in United Kingdom. BMJ, 314, 929-932.

Waddell, G. (2002) Models of Disability: Using Low Back Pain as an Example. London: Royal Society of Medicine Press.

M. Henderson Institute of Psychiatry, London, UK

C. Bass Department of Psychological Medicine (Barnes Unit), John Radcliffe Hospital, Headington, Oxford OX3 9DU, UK

J. Poole Dudley, Beacon \& Castle Primary Care Team, Dudley, UK

\section{Globalisation and biculturalism}

In their recent review article, Bhugra \& Mastrogianni (2004) describe the cultural and mental health aspects of what is now called globalisation, and its present and future impact upon mental disorders, with a special reference to depression. Among the many unknowns in that matter, the authors point towards the issue of whether cultures will homogenise, which seems improbable, or whether the tendency for communities to reassert their distinctive ethnic identities will prevail. Eventually, it seems reasonable to believe that different forms of equilibrium will develop between these apparently opposed forces, including what anthropologists call 'creolisation of cultures'. In that perspective, the issue of biculturalism deserves further elaboration.

Until recently, biculturalism was considered mainly in the perspective of partnership for ethnic minorities in a mainstream cultural environment. Different models of second-culture acquisition have been recognised and studied. In their classical work, LaFromboise et al (1993) reviewed typical patterns of biculturalism: the assimilation, acculturation, alternation, multicultural and fusion models. In that acceptance of biculturalism, the ideal goal for an individual 